\title{
In Situ Visualization of the Electron Wind Force in the Elastic Regime
}

Matthew Mecklenburg ${ }^{1}$, Brian Zutter $^{2}$, William A. Hubbard ${ }^{3}$, Xin Yi Ling ${ }^{3}$ and B. C. Regan ${ }^{4}$

${ }^{1}$ The Aerospace Corporation, United States, ${ }^{2}$ University of California, Los Angeles, Los Angeles, California, United States, ${ }^{3}$ University of California, Los Angeles, United States, ${ }^{4}$ Department of Physics and Astronomy, University of California, Los Angeles, California 90095, United States, United States

A flow of electrons in a wire forces the atomic nuclei from their equilibrium positions. A sufficiently large current density will break the metallic bonds binding the nuclei and induce an atomic current [1]. The atomic current can eventually move enough material (via electromigration) to form a void that breaks an electrical connection. However, preceding this breaking point an induced pressure gradient stabilizes the wire by producing a force density that opposes mass transport [2,3]. Mapping the bulk plasmon resonance energy (to 0.8 parts-per-thousand precision) in $1.1 \mu \mathrm{m}$ long aluminum nanowire, we exploit the plasmon energy's sensitivity to the wire's density to visualize the pressure gradient produced by the electron wind force. We achieve a pressure sensitivity of $50 \mathrm{MPa} / \sqrt{ } \mathrm{Hz}$ with sufficient spatial resolution to observe atomic features such as grain boundaries. The wire's density changes with the applied current because of temperature and pressure effects. These effects can be separated because the temperature $T$ [4] is even in the polarity of the applied current while the pressure $P$ is odd. A sequence of five spectrum images with zero, positive, zero, negative, and zero current, respectively, is sufficient to produce separate maps of the two thermodynamic state variables $T$ and $P$.

Using a scanning transmission electron microscope (STEM), we raster a $100 \mathrm{pA}, 1$-nm diameter electron beam across an electron-transparent, lithographically-defined aluminum wire supported by a 20 -nm thick silicon nitride membrane. We perform electron energy loss spectroscopy (EELS) at each beam position to form a spectrum image. We then fit both the zero-loss peak and the plasmon peak in each spectrum to extract precision measurements of the plasmon energy. The polycrystalline wire exhibits no signal in temperature or pressure that is correlated with its grain structure [5]. The temperature changes correspond to the expectation for Joule heating: the wire is warm in its middle and near room temperature at its ends, which are heat-sunk by the contacts.

In some ways the pressure also behaves as expected, in that it builds up linearly along the wire's length. However, rather than trailing off at the ends of the wire, where the current density drops precipitously, the pressure returns to zero more than $70 \mathrm{~nm}$ inside the wire, where the current density is still high. This unexpected indentation is not correlated with any features revealed by bright field and dark field STEM imaging. The pressure gradient is expected to be proportional to the current density, and the resulting expectation for the pressure distribution (Fig. 1, dashed red curve) does not match the observation. Likewise, the pressure attenuation rate is expected to depend on the rate at which the wire's cross-sectional area changes, but the measured pressure attenuation rate is $5 \times$ greater. (The slopes in the dashed line are determined using STEM EELS measurements of the wire's thickness along with standard STEM mapping

of its other dimensions.) These unexpected deviations from the elastic response are present in both serpentine and straight wires. 


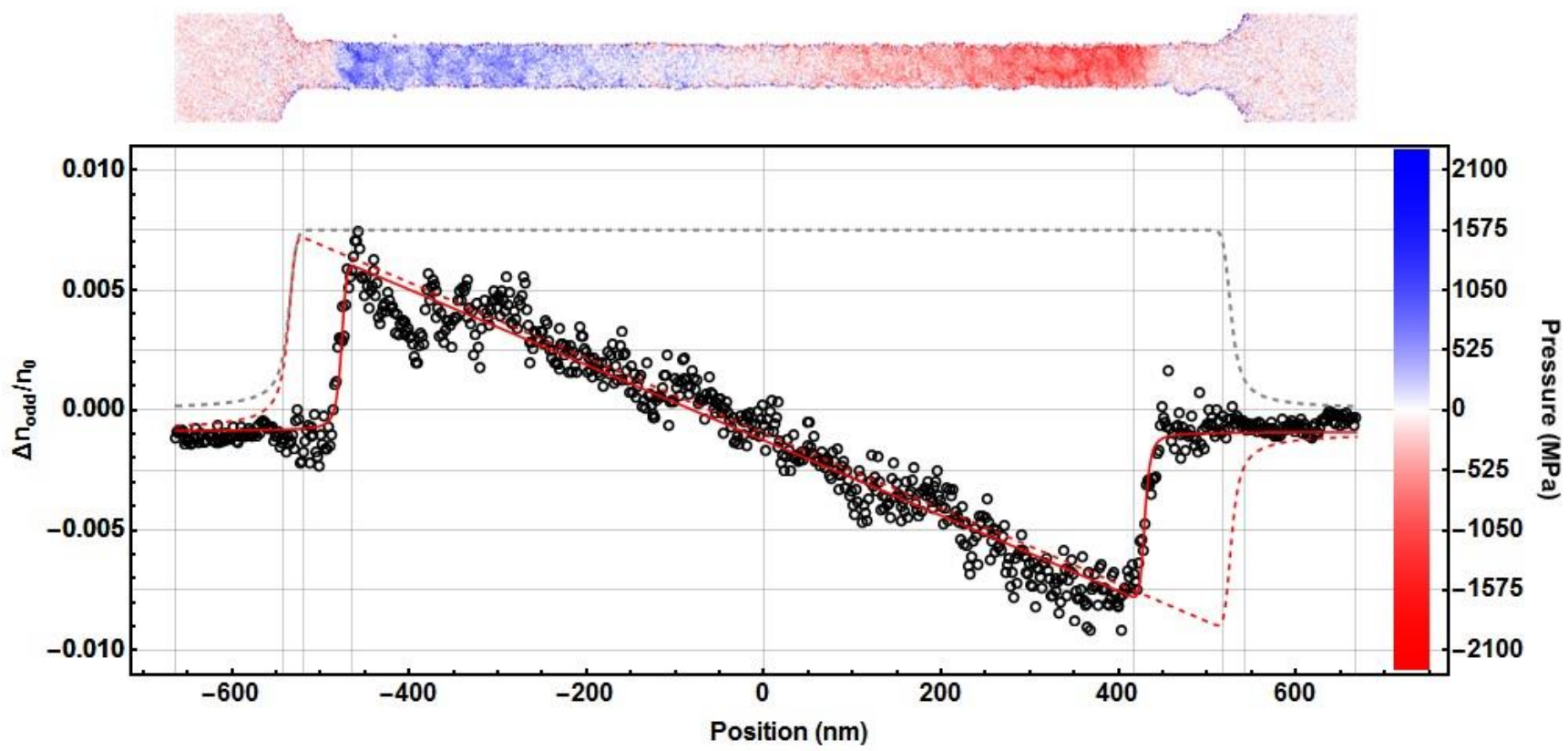

Figure 1. Figure 1. A pressure map of the aluminum wire (top) and the corresponding line profile (bottom). Aluminum's density (left axis) varies with the applied pressure $\mathrm{P}$ according to relations parametrized by its bulk modulus. Inverting these relations gives the local pressure (right axis). The data is fit (red solid curve) to an analytic function with the effective valence charge $Z^{*}$, the length of the wire $\mathrm{L}$, and the attenuation length scale $\ell$ as free parameters. The pressure expectation (dashed red curve) has $L$ and $\ell$ fixed by the wire's measured dimensions. Similar pressure profiles occur in serpentine wires. Interestingly, the pressure effect, like the temperature effect, is insensitive to curvature in the wire: it is unimpeded by corners. The dashed grey line is a scaled current density plot. It shows how the current density decreases rapidly in the contacts, highlighting the unexpected spatial separation between the current density drop and the pressure drop.

References

[1] J Lienig and M Thiele, "Fundamentals of Electromigration-Aware Integrated Circuit Design" (Springer International Publishing, Cham). 2018

[2] I A Blech, Electromigration in thin aluminum films on titanium nitride, Journal of applied physics 47, 1203-1208 (1976)..

[3] I A Blech and C Herring. Stress generation by electromigration. Applied Physics Letters, 29, 131-133 (1976).

[4] M Mecklenburg et al., Science 347 (2015), p. 629-632.

[5] C. Witt, Electromigration in Bamboo Aluminum Interconnects, Ph.D. thesis, Stuttgart, Stuttgart (2000).

[6] This work was supported by the Semiconductor Research Corporation (SRC), by National Science Foundation (NSF) awards DMR-1611036 and DMR-2004897, by NSF STC award DMR-1548924, and by The Aerospace Corporation's SERPA program. 